\title{
Computer Model for Determination of the Physiologic Correlates of the Impedance Cardiovasculogram Associated with Acute Heart Failure
}

\author{
RL Summers \\ University of Mississippi Medical Center, Jackson, MS, USA
}

\begin{abstract}
The cardiovasculogram (CVG) is the graphical depiction of the electronic signals generated by the technique of impedance cardiography (ICG) by measurement of the mechanical function of the cardiac cycle. Previous studies have suggested that the presence of a prominent " $O$ " wave in the CVG may be a sensitive indicator of fluid volume overload during episodes of decompensated heart failure. In this study, a computer model of a circulatory system interfaced with a simulated bioimpedance technology is used to determine which physiologic elements may be associated with the typical changes seen in the $C V G$ during acute heart failure.
\end{abstract}

\section{Introduction}

Originally, electrocardiography was primarily used to measure and monitor the rate and rhythm of the heart. Eventually, clinicians began to realize the wealth of information available from an analysis of the structural changes of the electrocardiogram (ECG) waveforms. In current medical practice, the changes seen in the constructs of the ECG waveforms are used in the diagnosis of such common clinical conditions as acute myocardial infarction and left ventricular hypertrophy. While the ECG describes the electrical events of the heart, a similar analysis of the impedance derived cardiovasculogram (CVG) waveforms provides information concerning the mechanical events of the heart [1]. The CVG waveform is rich in information concerning systolic contractility, diastolic and atrial functioning and systemic venous return. Characteristic deviations from the structure of the typical CVG waveform have been correlated with a variety of commonplace cardiovascular pathologies [2,3,4,5]. There is also evidence that the presence of such characteristic CVG waveforms patterns may be useful in the diagnoses of pathologic states such as congestive heart failure, mitral regurgitation and IHSS.
The form of the CVG can be static (as with congenital heart diseases) or time-varying as the clinical condition progresses or improves (as with acute decompensated heart failure). For instance, because we know the $\mathrm{O}$ wave reflects the diastolic period of the cardiac cycle then it is easy to conclude that dramatic changes in the contour and size of this waveform indicates an abnormality in the process of movement of blood from the aorta to the vena cava and the venous return to the heart. If the heart is failing, there is a rise in atrial pressures and congestion within the venous side of the circulation. This increase in the amount of blood within the highly compliant vena cava would be expected to produce a reduction in impedance to flow of the ICG current and a larger deflection of the diastolic $\mathrm{O}$ wave as fluid moves in and out of the venous side of the circulation $[5,6,7]$.

The art of "reading the ECG" has become an essential skill for all modern day clinicians. The field of CVG waveform analysis is relatively new and evidentiary information concerning archetypal waveforms is lacking for the wide variety of possible pathologies seen in typical medical practice. These archetypal CVG profiles have only recently been used clinically in the differentiation and monitoring of their respective disease states [2]. In this study, a computer model of circulatory physiology interfaced with a simulated ICG technology was used as a methodology for the determination of the physiologic correlates of the CVG waveform characteristics associated with acute heart failure. Such a methodology has the potential to assist clinicians in the development of additional archetypal CVG profiles.

\section{Methods}

An anatomically structured computer model of a circulatory system interfaced with simulated bioimpedance technology was constructed using a graphic-based simulation software (VisSim, Inc.). The circulatory model is comprised of separate systemic 
arterial and venous compartments as well cardiopulmonary components. The model dynamics function in a typical Windkissel framework. There are a total of 68 variables involved in 991 interactions within the model. A standard Euler numerical methodology is used in the solution. The model and support software is capable of running dynamic simulations of the circulatory physiology of the cardiac cycle while generating the resultant predicted impedance derived CVG waveform (Väsamed® methodology).

Simulations of the conditions of acute heart failure (systolic and diastolic) were performed by altering the model parameters controlling ventricular contractility and compliance ( $1 / 2$ normal values). For model validation, simulated changes in the $\mathrm{O}$ and $\mathrm{C}$ waveforms with respect to height and breadth were compared to $\mathrm{CVG}$ changes in patients with both systolic and diastolic acute heart failure (figure 1). A systems analysis of the model was performed to determine the physiologic mechanisms responsible for the observed CVG changes.
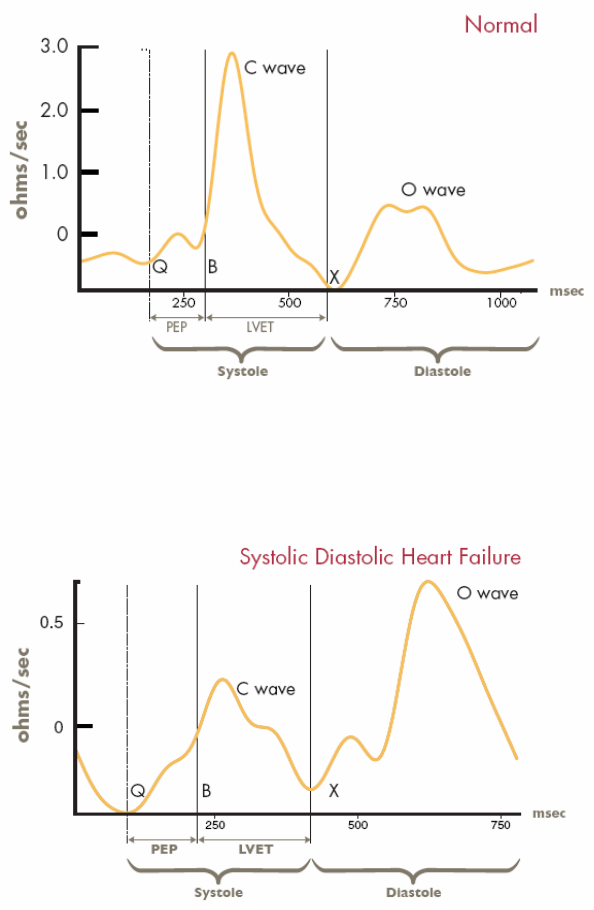

Figure 1. CVG waveforms of the normal and failing heart: Q: Beginning of the QRS complex of the ECG; B: aortic valve opening; $\mathrm{C}$ : maximum aortic flow; $\mathrm{X}$ : aortic valve closing; O: mitral valve opening; PEP: pre-ejection period; LVET: left ventricular ejection time.

\section{Results}

Figure 2 compares the overlapping CVG results of a simulation of a normal patient (A), a patient with systolic dysfunction, and a patient with diastolic dysfunction (C). After a reduction in the model's cardiac contractility, the simulated CVG had a 2 fold increase in the peak of the $\mathrm{O}$ wave with a broadening and blunting of the systolic $\mathrm{C}$ wave as compared to the normal CVG. The simulation study of diastolic heart failure resulted in a wide and elevated $\mathrm{O}$ wave with mild changes in $\mathrm{C}$ wave morphology. The systems analysis of the model in the context of these simulated conditions of heart failure revealed that congestive changes resulting in increases in sequestered fluid volumes in the vena cava were responsible for the characteristic changes in the $\mathrm{O}$ waveform. When this venous sequestration of blood was prevented by an amelioration of the fluid overload or by artificially fixing venous compliance in the computer model, the $\mathrm{O}$ wave changes were much less prominent and resembled the normal CVG.

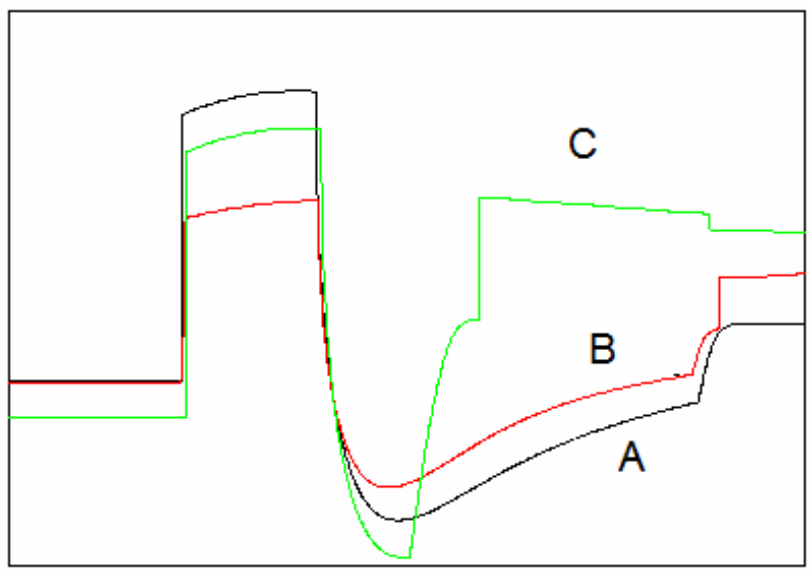

Figure 2. Overlapping simulated CVG waveforms of the normal and failing heart: A: normal patient; B: patient with systolic dysfunction; $\mathrm{C}$ : patient with diastolic dysfunction.

\section{Discussion and conclusions}

The primary purpose of this study was to examine the feasibility of using a computer model of cardiovascular physiology to determine the physiologic correlates of the characteristic patterns typically seen in the waveform of the impedance CVG of a patient with acute heart failure. If specific CVG structural elements are found to correspond with known pathophysiologic derangements of heart failure, then an identification of these waveform characteristics might be useful in the diagnosis and monitoring of patients with these conditions. In this 
effort a specialized computer model incorporating the features of an impedance cardiographic monitor was developed for the analysis of the impedance derived $\mathrm{CVG}$ during a simulation acute heart failure.

For validation purposes, the model's graphical output was first compared to the CVG waveforms obtained from patients in acute heart failure. While it is evident that the structural features of the typical CVG can be identified from the simulation graphic, this model-based depiction is coarse in comparison to the patient derived measurements. Further refinement of the model to include greater compartmentalization and a finer integration step size would provide better resolution. However, general changes in the $\mathrm{C}$ and $\mathrm{O}$ waveforms of the heart failure simulated CVG were found to be typical of those seen in patients with this condition.

A systems analysis performed using the model indicates that fluid congestion within the vena cava typically seen in patients with acute heart failure may be responsible for these characteristic waveform changes. This analysis suggests that the typical CVG pattern of acute heart failure has a specific physiologic etiology and that the examination of certain waveform elements can potentially be used for monitoring the response to treatment in these patients. A clinical response to diuresis would be reflected in a resolution of the abnormal O wave changes [8].

This study indicates that computer modeling of the circulatory-bioimpedance interface may lead to a more detailed understanding of the physiologic and pathophysiologic meaning of the CVG waveforms. This methodology could be extremely importantly in the development of archetypal CVG profiles from which clinicians might perform pattern recognition of classic pathophysiologic states [8].

\section{Acknowledgements}

I would like to acknowledge the assistance of Väsamed, Inc.

\section{References}

[1] Summers RL, Shoemaker W, Peacock WF, Ander. Bench To Bedside: Electrophysiologic and Clinical Principles of Noninvasive Hemodynamic Monitoring Using Impedance Cardiography. Acad Emerg Med 2003; 10:669-680.

[2] Summers RL, Kolb JC, Woodward LH, Galli RL. Differentiating systolic from diastolic heart failure using impedance cardiography. Acad Emerg Med 1999;6:693-9S

[3] Lababidi Z, Ehmke DA, Durnin RE, Leaverton PE, Lauer RM. The first derivative thoracic impedance cardiogram. Circulation 1970; 41:651-658.

[4] Bhardwaj PR, Jindal GD, Dharani JB, Parulkar GB. Impedance cardiography in valvular diseases of the heart. Indian Heart J. 1983;35:46-9.

[5] Woltjer HH., Bogaard HJ., Bronzwaer JG.,de Cock CC., de Vries PM. Prediction of pulmonary capillary wedge pressure and assessment of stroke volume by noninvasive impedance cardiography. Am Heart J 1997; 134:450-455.

[6] Summers RL. Emerging Diagnostics: Impedance Cardiography in the assessment and management of acute heart failure. Crit Path in Card. 2005; 4:134-139.

[7] Summers RL, Cardiovascular Emergencies: Chapter 32: Future Diagnostics: Impedance Cardiography in the Assessment and Management of Acute Heart Failure 2006; McGraw-Hill Companies New York, NY pp 357-363.

[8] Summers RL, CVG Waveform Atlas: Heart Failure Patient Profiles. 2006 C Vasamed Corp, Eden Prairie, Minnesota.

Address for correspondence:

Richard L. Summers, MD

Department of Emergency Medicine

University of Mississippi Medical Center

2500 North State Street

Jackson, MS 39216

E-mail: rsummers@pol.net 